\title{
Study on Asymmetric Magnetic Pole Structure for IPM Motor Using Neodymium Bonded Magnet
}

\author{
T. Yanagisawa, Y. Yoshida*, and K. Tajima \\ Department of Cooperative Major in Life Cycle Design Engineering, Akita Univ., 1-1, Tegata Gakuen-machi, Akita 010-8502, \\ Japan \\ *Department of Mathematical Science and Electrical-Electronic-Computer Engineering, Akita Univ., 1-1, Tegata Gakuen-machi, \\ Akita 010-8502, Japan
}

In this paper, we propose an interior permanent magnet synchronous motor (IPMSM) with an asymmetric magnetic pole structure that uses bonded neodymium magnets. By adopting the asymmetrical magnet arrangement, the current phase angle, where the magnetic torque is maximized, can be set to almost the same phase while maximizing the reluctance torque. To verify the usefulness of the proposed motor, we prototyped an actual motor on the basis of our proposed motor and compared the characteristics with a conventional IPMSM that uses sintered neodymium magnets. Although the torque of the proposed motor was smaller than the conventional motor due to a problem with the dimensions of the bonded neodymium magnets, it was shown that an IPMSM with an asymmetrical magnetic pole structure that uses Dy-free bonded neodymium magnets can achieve performance equivalent to conventional IPMSMs that use sintered neodymium magnets.

Keywords: permanent magnet synchronous motor, interior permanent magnet synchronous motor, neodymium bonded magnet, asymmetric pole structure, heavy rare earth free

\section{Introduction}

In recent years, interest in protecting the global environment and conserving energy has been growing. Among electric motors, permanent magnet synchronous motors (IPMSMs) using high-performance rare earth magnets for high-efficiency motors have been applied to various fields. Neodymium (Nd) and dysprosium (Dy) are key elements for improving the performance of PMSMs; however, the production of Dy, a heavy rare-earth element, depends on specific countries. Therefore, developing high-efficiency motors without using Dy is required.

Although neodymium bonded magnets have a lower residual magnetic flux density than neodymium sintered magnets, they are expected to be applied to motors as Dy-free magnets because of their advantages, such as a stronger residual magnetic flux density than ferrite magnets, high degree of freedom in terms of shape, and zero generation of eddy currents.

Since IPMSMs can utilize reluctance torque in addition to magnetic torque, a higher torque can be expected compared with PMSMs that do not utilize reluctance torque. Although it has been reported that reluctance torque is used to achieve the same output characteristics, power density, and efficiency characteristics as rare earth magnets even when ferrite magnets are used ${ }^{1}$, it is difficult to produce a torque density equivalent to that of a rare earth magnet motor only with a ferrite magnet. In examining the optimum shape of an IPMSM using a rare-earth bonded magnet, a torque equivalent to that of rare earth sintered magnet IPMSMs has been realized, but the rotor has an overhang structure, and there are still problems with further downsizing these IPMSMs ${ }^{2}$.

Generally, an IPMSM has a phase difference of 45 degrees between the current phase angle at which the magnetic torque is maximized and the current phase angle at which the reluctance torque is maximized. Therefore, there has been study on improving the total torque by arranging the magnets asymmetrically and shifting the phase of the magnetic torque ${ }^{3)-6)}$. In asymmetric rotor designs for the permanent magnet-assisted synchronous reluctance motor, the total torque was increased by designing the rotor to have an asymmetric structure, but the reluctance torque was reduced compared with the conventional structure $^{4), 6)}$.

In this study, we design and prototype an asymmetric pole rotor for an IPM motor with improved reluctance torque compared with the previous model studied ${ }^{7)}$

\section{Principle of improving torque with asymmetric pole structure}

Here, we use a 2-pole inset-type PMSM rotor having saliency to explain the principle of improving the torque with the asymmetric pole structure. Fig. 1 (a) and (b) show the torque generation principle of an inset-type PMSM. The center of the north pole of the magnet is the $d$-axis, and the direction advanced 90 degrees from the $d$-axis in the rotational direction is the $q$-axis. The magnetic torque generated by suction and repulsion forces acting between the pole of the rotating magnetic field and the pole of the permanent magnet placed on the rotor is maximized by the magnetic field rotating in the $q$-axis direction as shown in Fig. 1 (a). The reluctance torque that is generated by the suction force acting between the salient pole of the rotor and the rotating magnetic field is maximized by the magnetic field rotating in the direction advanced 45 degrees from the $q$-axis as shown in Fig. 1 (b). Therefore, the maximum value of both torque components cannot be generated simultaneously.

To generate the maximum value of these two 
torque components simultaneously, the phase of the magnets is advanced without changing the phase of the rotor saliency shown as Fig. 2. In Fig. 2, the magnets are divided and arranged in a phase-shifted manner, while the phase of the rotor saliency remains the same as the conventional inset-type PMSM as shown in Fig. 1 (a). If the phase shift of the magnet poles can be advanced 45 degrees from the $q$ axis direction, the maximum torque of the two components can be generated at the same phase, and the total torque can be improved.

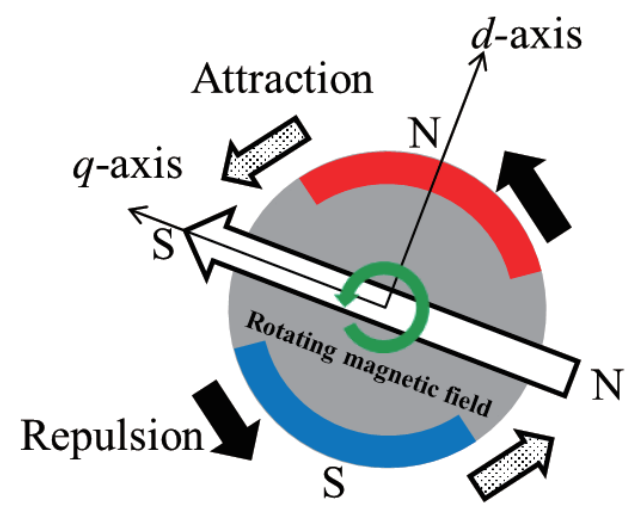

(a) Magnetic torque

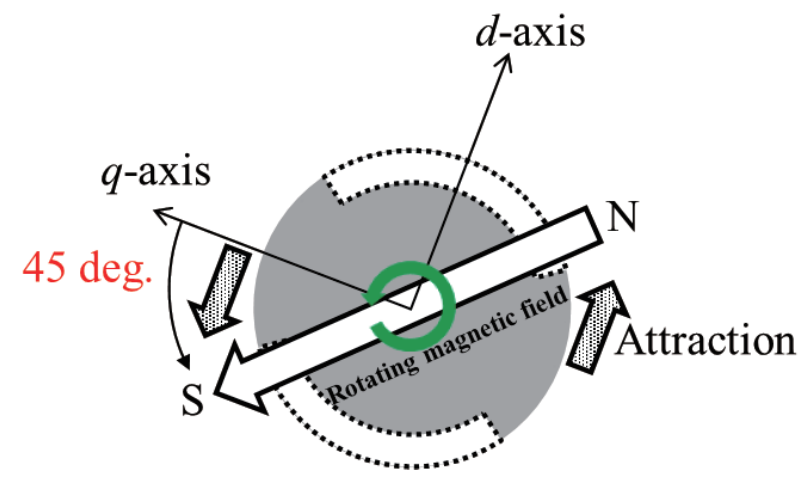

(b) Reluctance torque

Fig. 1. Principle of torque generation of 2-pole inset-type PMSM

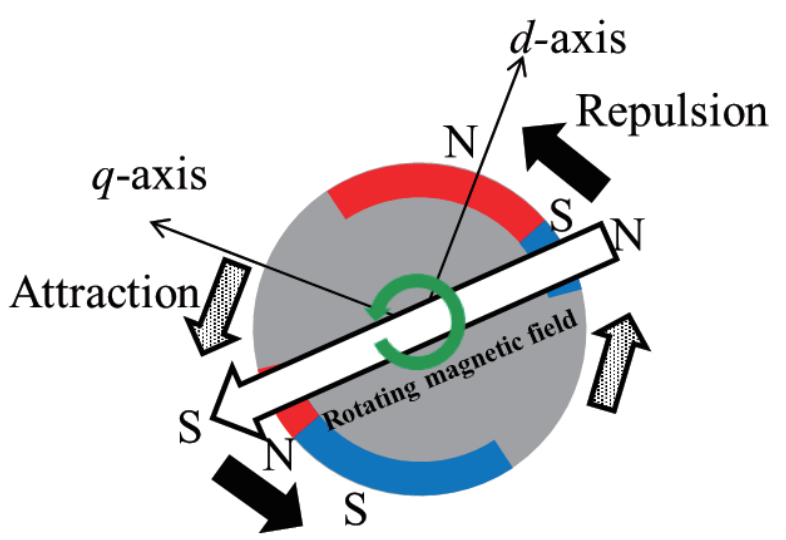

Fig. 2. Principle of magnetic torque generation when magnets are divided and arranged in phase-shifted manner

\section{Analysis of examined motors}

\subsection{Overview of examined motors}

Fig. 3 (a) shows the conventional IPMSM with 4-pole sintered neodymium magnets (base model), and Fig. 3 (b) shows the proposed IPMSM with a 4-pole asymmetric rotor structure using bonded neodymium magnets. The stator is common to both models. Table 1 shows the motor specifications. The residual magnetic flux density of the sintered neodymium magnets used in the base model and bonded neodymium magnets used in the proposed model are $1.27 \mathrm{~T}$ and $0.71 \mathrm{~T}$, respectively.

Fig. 4 (a) and (b) show the rotor shapes and magnetization directions of the base and proposed models. The void arrows in the figure indicate the magnetization direction of the magnet. The part highlighted on the rotor surface with the solid line represents the $\mathrm{N}$ pole, and that highlighted with the broken line represents the $\mathrm{S}$ pole. The magnetization direction of the base model faces the same direction as the $d$-axis direction. In the rotor of the proposed model, since the magnets are arranged asymmetrically, the magnetic pole center exists at a position shifted from the $d$-axis direction.

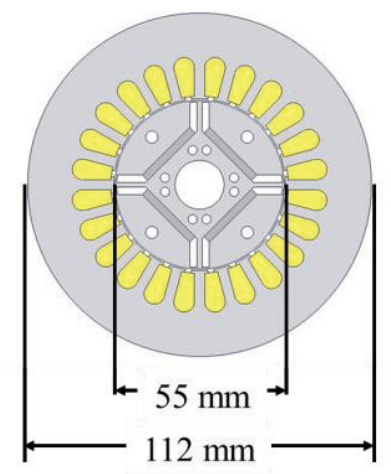

(a) Base model

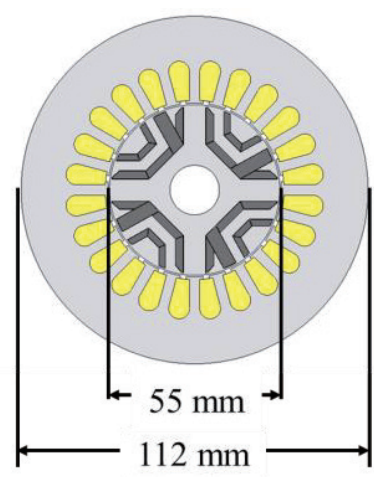

(b) Proposed model
Fig. 3. Shape of motors under consideration

Table1 Specifications of analytical models

\begin{tabular}{|c|c|c|}
\hline Parameter & Base & Proposed \\
\hline Permanent magnet material & $\begin{array}{c}\text { Neodymium } \\
\text { sintered }\end{array}$ & $\begin{array}{c}\text { Neodymium } \\
\text { bonded }\end{array}$ \\
\hline Core material & \multicolumn{2}{|c|}{50 A350 } \\
\hline Coil & \multicolumn{2}{|c|}{ Copper } \\
\hline Stator outer diameter $(\mathrm{mm})$ & \multicolumn{2}{|c|}{112} \\
\hline Rotor outer diameter $(\mathrm{mm})$ & \multicolumn{2}{|c|}{55} \\
\hline Gap width $(\mathrm{mm})$ & \multicolumn{2}{|c|}{0.5} \\
\hline Stack length $(\mathrm{mm})$ & \multicolumn{2}{|c|}{30} \\
\hline Number of winding turns/slot & \multicolumn{2}{|c|}{24} \\
\hline Number of slots & \multicolumn{2}{|c|}{0.71} \\
\hline Number of poles & 1.27 & 470 \\
\hline Residual flux density $B_{r}(\mathrm{~T})$ & 876 & 1.20 \\
\hline Coercive force $H_{c}(\mathrm{kA} / \mathrm{m})$ & 1.05 & \\
\hline Recoil permeability $\mu_{r}$ & \multicolumn{2}{|c|}{} \\
\hline
\end{tabular}




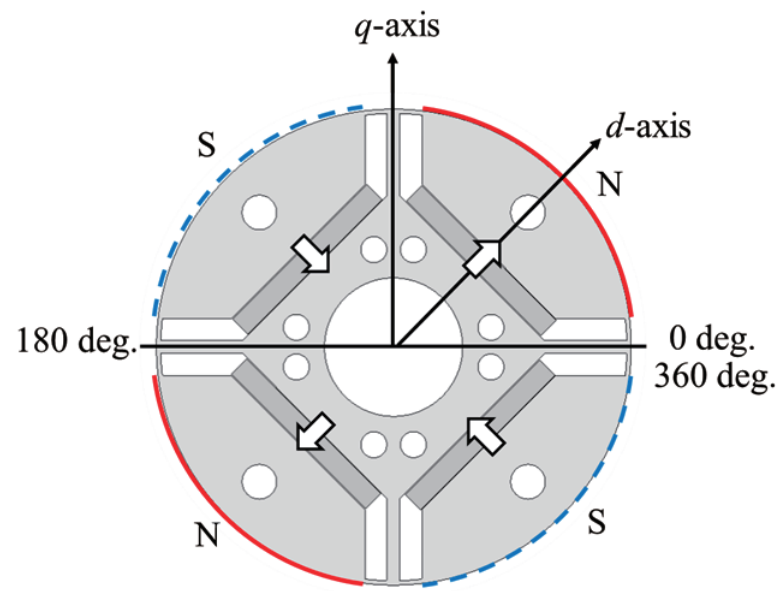

(a) Base model

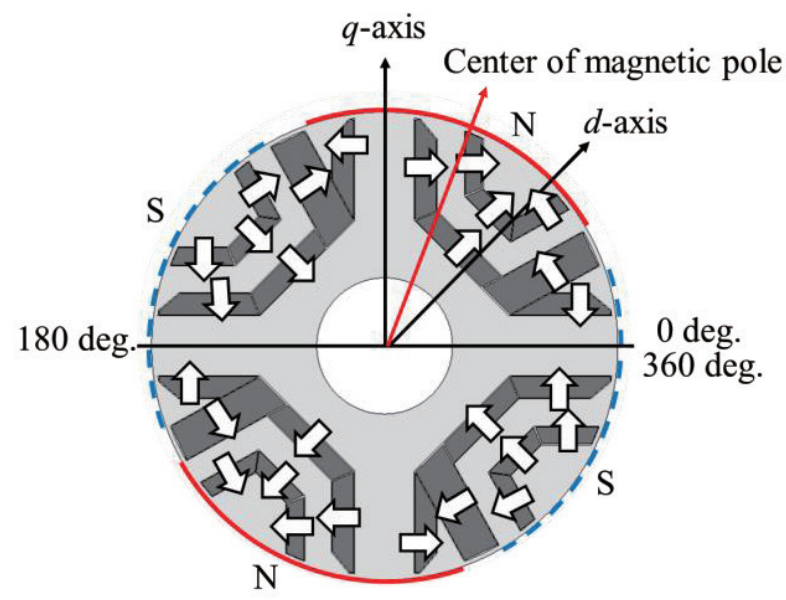

(b) Proposed model

Fig. 4. Magnetization direction

\subsection{Comparison of torque characteristics}

Fig. 5 shows the torque characteristics of the examined motors. The magnetic torque is indicated by the broken line, and the reluctance torque is indicated by the dotted line. The solid line is the total torque, that is, the sum of the magnetic torque and reluctance torque. The maximum magnetic torque of the base model was $0.85 \mathrm{~N} \cdot \mathrm{m}$ when the current phase angle $b$ was 0 degrees, and the maximum value of the reluctance torque was $0.20 \mathrm{~N} \cdot \mathrm{m}$ when the current phase angle $B$ was 45 and -135 degrees. The maximum magnetic torque of the proposed model was $0.77 \mathrm{~N} \cdot \mathrm{m}$ when the current phase angle $B$ was 40 degrees, and the maximum reluctance torque was $0.18 \mathrm{~N} \cdot \mathrm{m}$ when the current phase angle $B$ was 50 and -140 degrees. Therefore, the phase of the magnetic torque of the proposed model was shifted by 40 degrees compared with the base model, and it was confirmed that the current phases at which the magnetic and reluctance torques were maximum were almost the same. Comparing the maximum values of the total torque, the base model was $0.92 \mathrm{~N} \cdot \mathrm{m}$ when the current phase angle $B$ was 20 degrees, and that of the proposed model was $0.94 \mathrm{~N} \cdot \mathrm{m}$ when the current phase angle $B$ was 45 degrees. The proposed model obtained a maximum total torque equivalent to the base model when the phase of the magnetic torque was shifted by 40 degrees.

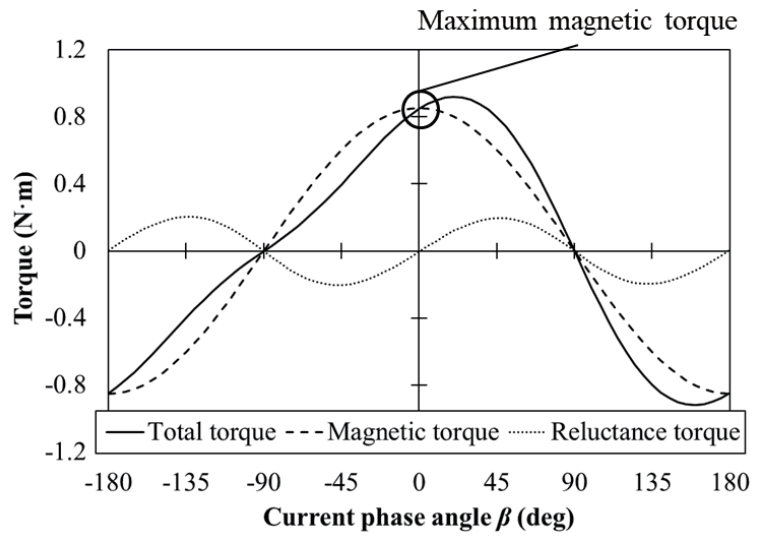

(a) Base model

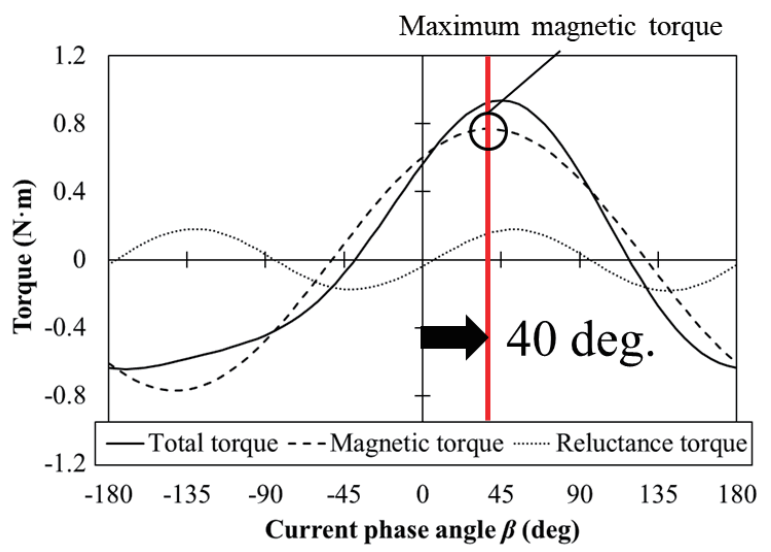

(b) Proposed model

Fig. 5. Torque characteristics of examined motors

\section{Experimental verification of motor performance}

\subsection{Experimental setup}

Fig. 6 shows photographs of a prototype stator and rotors used in an experiment. The same stator was used to measure the characteristics of the two motors. Fig. 7 shows a photograph of the experimental apparatus. The waveform of back electromotive force (EMF) was recorded by using a memory high coder. The input electric power of the motor $P_{\text {in }}$ was measured by using a power meter connected just before the motor, and the mechanical output power of the motor Pout was obtained from the product of the average torque $T$ and the rotational angular velocity $\omega$ measured by the torque detector shown in the figure.

\subsection{Comparison of calculated and measured values}

Fig. 8 (a) shows the back EMF waveform of the base model at $1300 \mathrm{rpm}$, and Fig. 8 (b) shows that of the proposed model. In the figures, the measured and calculated values are compared with each other.

The fundamental wave component was extracted from these waveforms with a fast Fourier transform (FFT) The relationship between the rotational speed and the fundamental component of the back EMF is shown in Fig. 9; Fig. 9 (a) is the base model, and Fig. 9 (b) is the proposed model. Comparing the calculated and measured values at $1300 \mathrm{rpm}$, the experimental value of the base model was $6.17 \%$ smaller than the calculated value, and the experimental value of the 
proposed model was $17.9 \%$ smaller than the calculated value. The reason for the larger error for the proposed model is that there were gaps between the magnet and the iron core greater than the designed value because of variation in the prototype magnet dimensions.

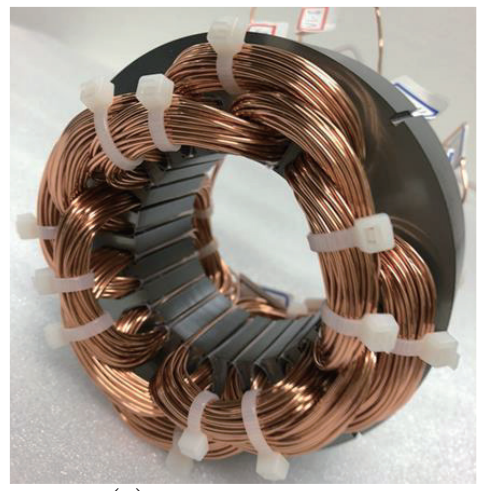

(a) Common stator
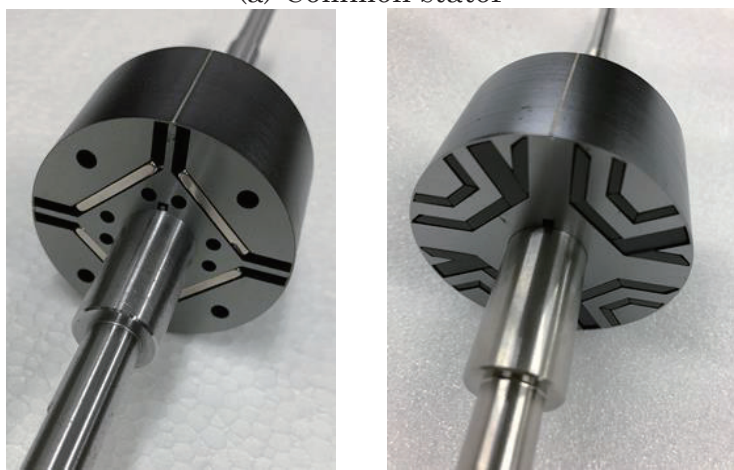

(b) Rotor (Base model)

(c) Rotor (Proposed model)

Fig. 6. Actual machines

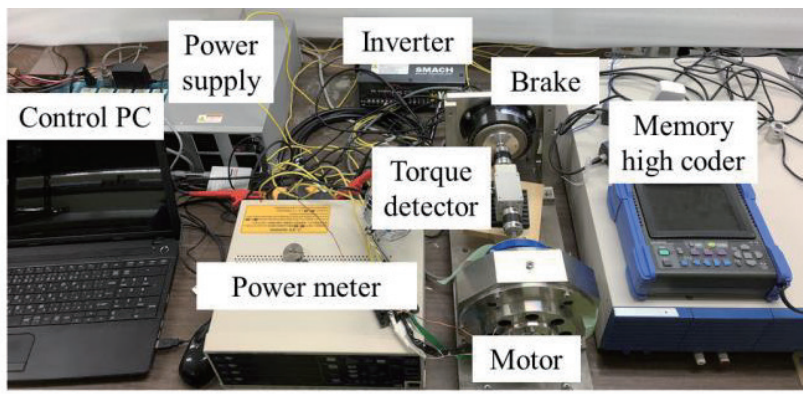

Fig. 7. Experimental apparatus

Fig. 10 shows the relationship between the current amplitude and torque of the base and proposed models at rotation speeds of 500, 1000, and $1300 \mathrm{rpm}$. The calculated values are indicated by the solid lines, and the measured values are indicated by the symbols. The current amplitude used for the horizontal axis of the graphs was the fundamental wave amplitude extracted by FFT from a current waveform including harmonic components as shown in Fig. 11. The current phase was selected so that the output torque was maximized for each current amplitude. From Fig. 10, the calculated value of the torque of the base model almost agreed with the experimental value at all rotational speeds, while the calculated value of the proposed model was larger than the experimental value. These results also

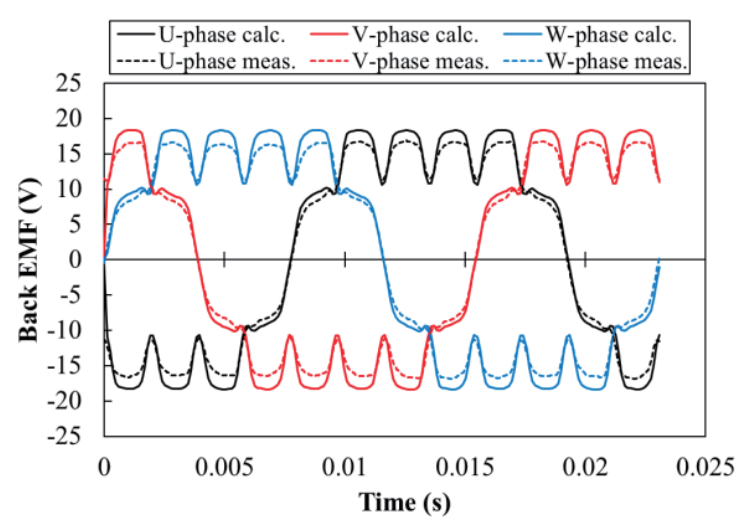

(a) Base model

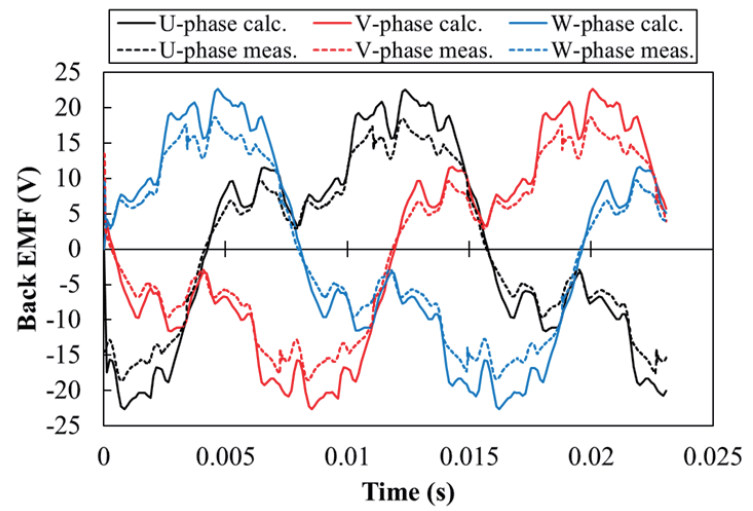

(b) Proposed model

Fig. 8. Back EMF waveform at 1300 rpm

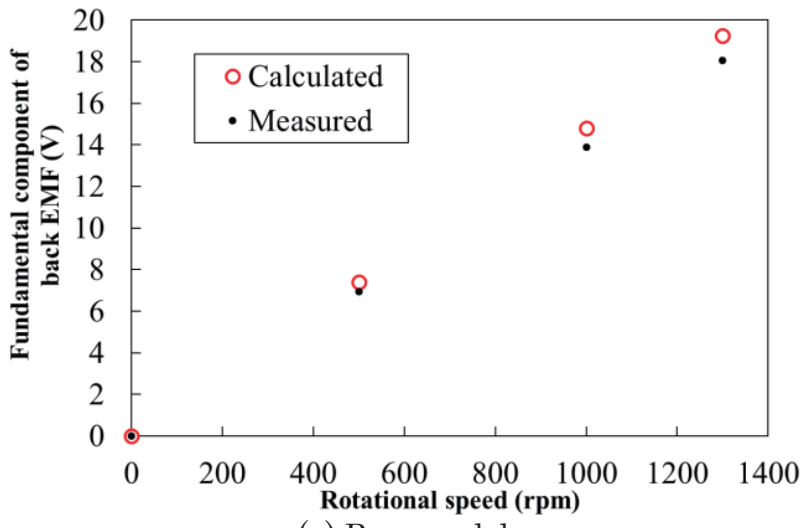

(a) Base model

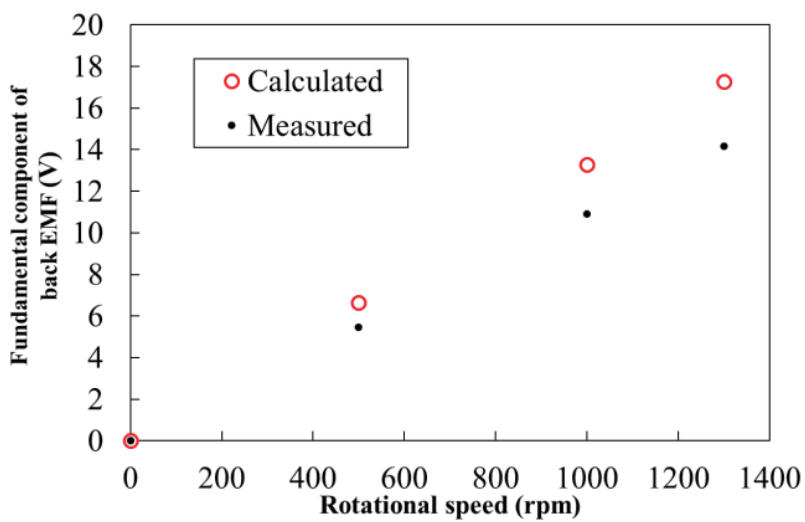

(b) Proposed model

Fig. 9. Relationship between rotational speed and fundamental component of back EMF. 
show that the proposed model could not be prototyped as designed due to variation in the magnet dimensions. It was shown that an asymmetrical magnetic pole structure for an IPMSM using Dy-free bonded neodymium magnets could achieve performance equivalent to the conventional IPMSM using sintered neodymium magnets if we can eliminate the error of the dimensions.

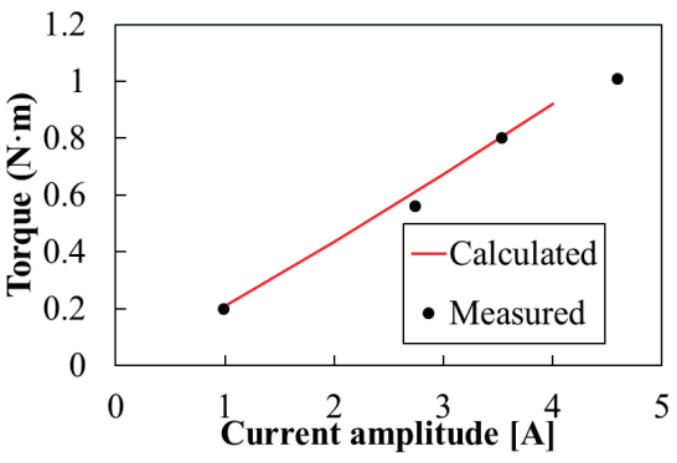

(a) Base model at rotational speed of $500 \mathrm{rpm}$

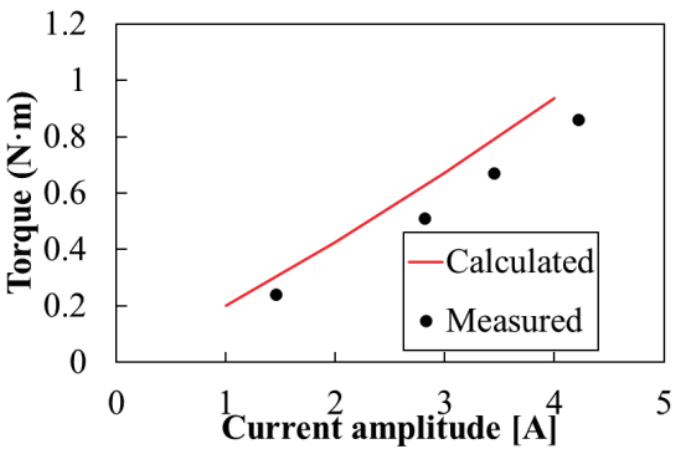

(b) Proposed model at rotational speed of $500 \mathrm{rpm}$

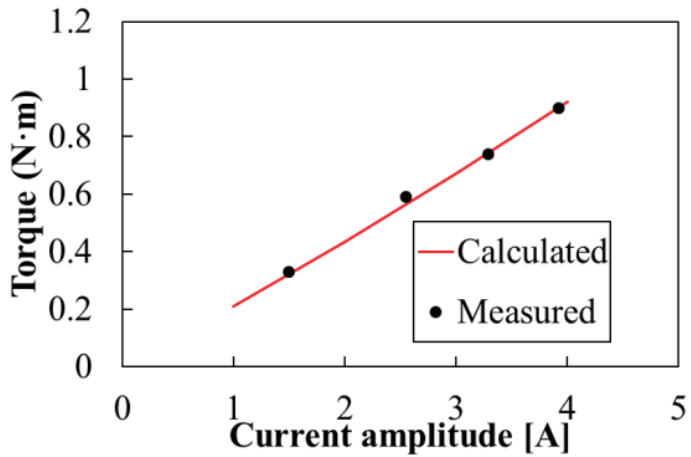

(c) Base model at rotational speed of $1000 \mathrm{rpm}$

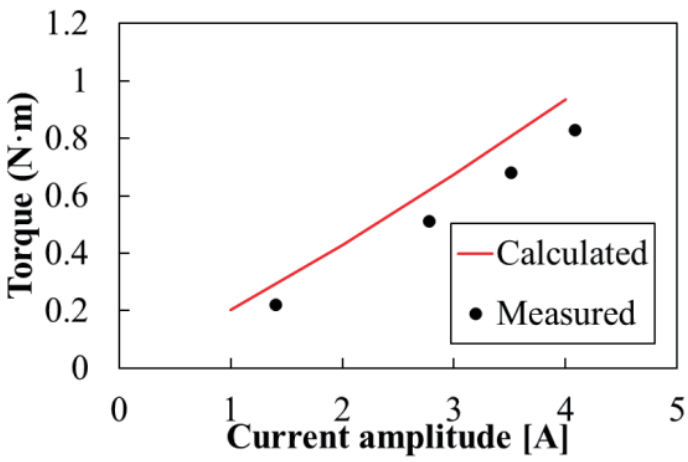

(d) Proposed model at rotational speed of $1000 \mathrm{rpm}$

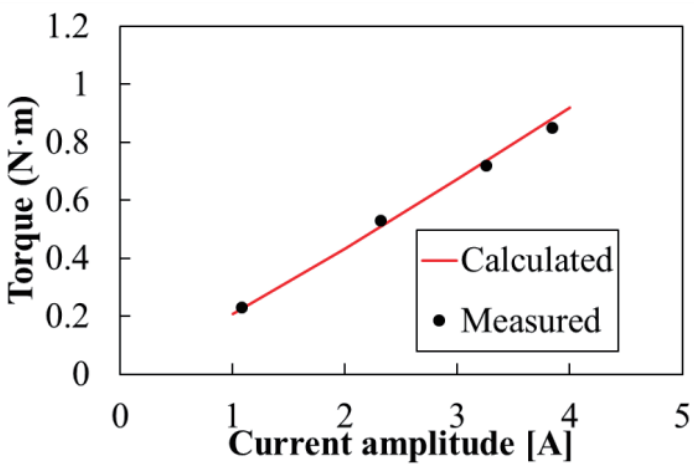

(e) Base model at rotational speed of $1300 \mathrm{rpm}$

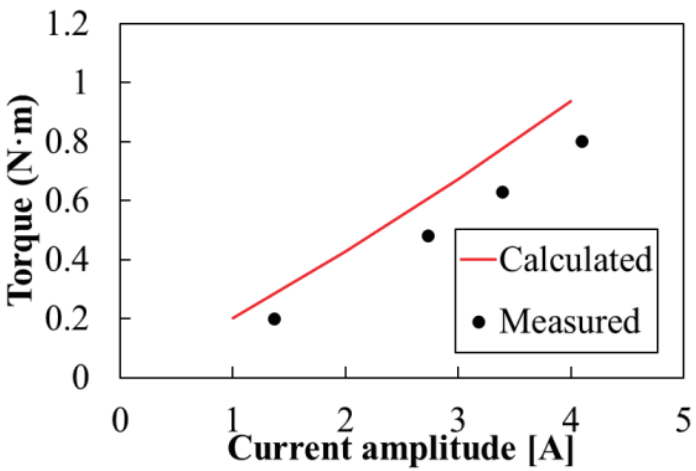

(f) Proposed model at rotational speed of $1300 \mathrm{rpm}$.

Fig. 10. Relationship between current amplitude and torque

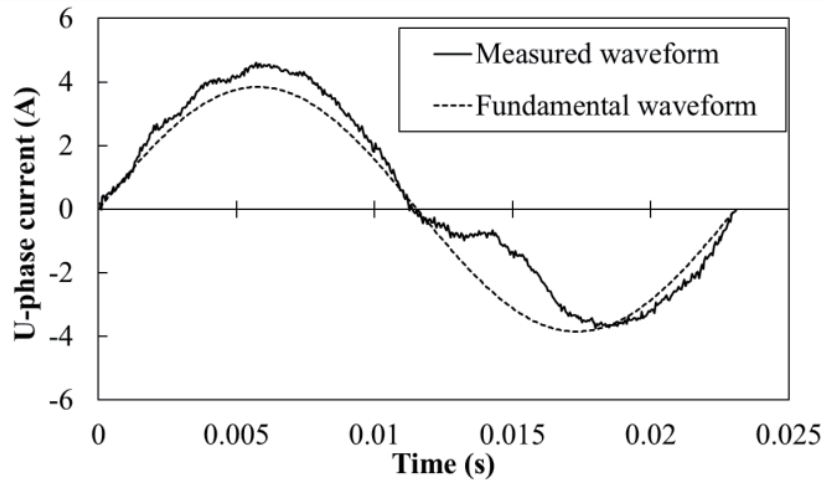

(a) Base model

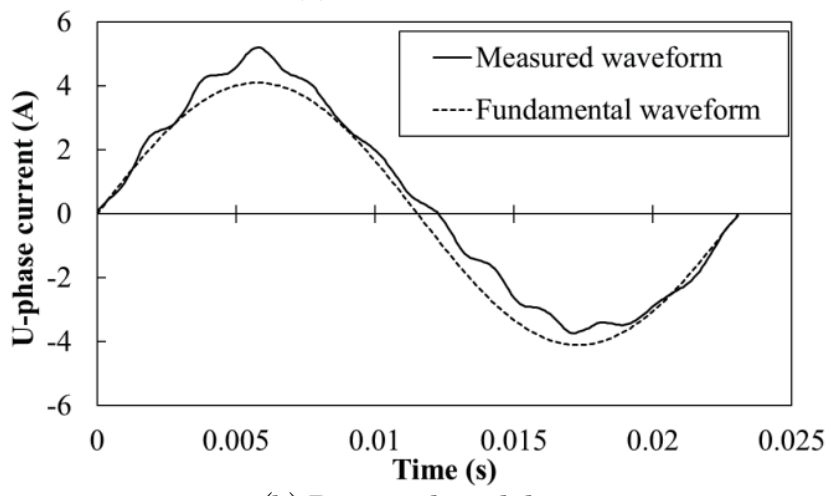

(b) Proposed model

Fig. 11. U-phase current waveform at maximum torque at $1300 \mathrm{rpm}$ 


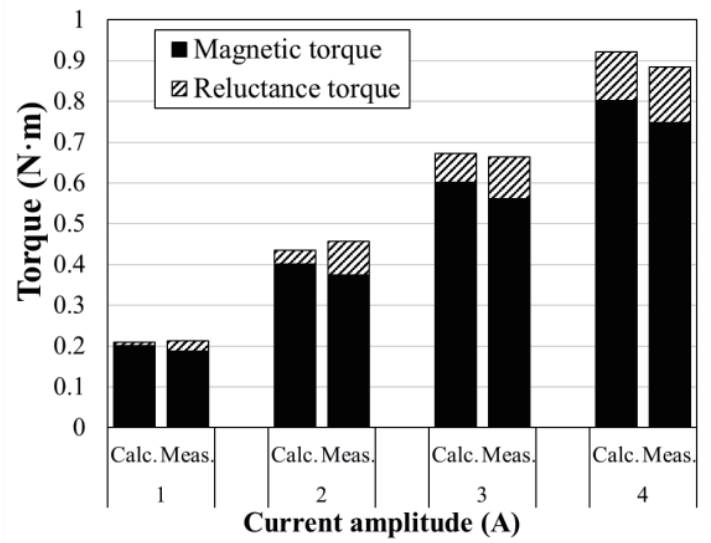

(a) Base model

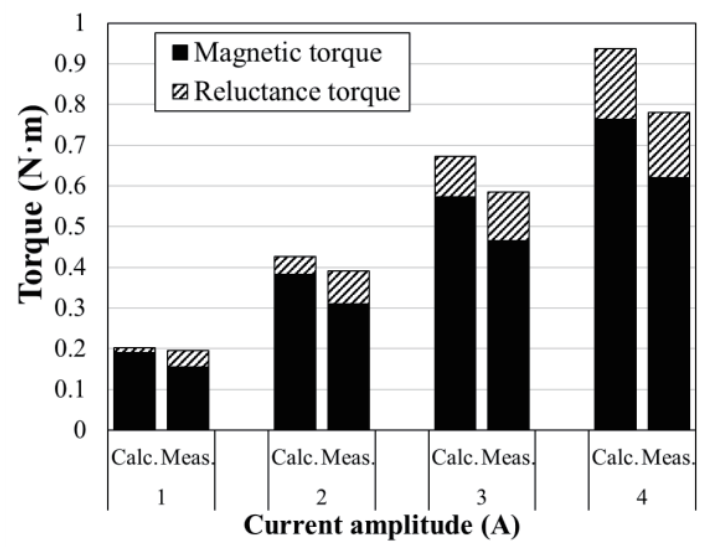

(b) Proposed model

Fig. 12. Relationship between current amplitude and separated torque components at $1300 \mathrm{rpm}$

Fig. 12 shows the relationship between current amplitude and separated torque components at 1300 rpm. The components were separated by using the following equations.

$$
\begin{gathered}
\Psi_{a}=\frac{V_{0}}{\omega} \\
T_{m}=P_{n} \Psi_{a} i_{q} \\
T_{r}=T-T_{m}
\end{gathered}
$$

Here, $V_{0}, \omega, P_{n}, \Psi_{a}, i_{q}, T_{m}, T_{r}$, and $T$ are values converted by the $d^{-} q$ transformation of the back EMF, angular velocity, number of pole pairs, magnetic flux of magnets, $q$-axis current, magnetic torque, reluctance torque, and total torque obtained in the experiments and calculation. The magnetic torque was calculated by multiplying $\Psi_{a}, P_{n}$ and $i_{q}$. The reluctance torque was calculated by subtracting $T_{m}$ from $T$.

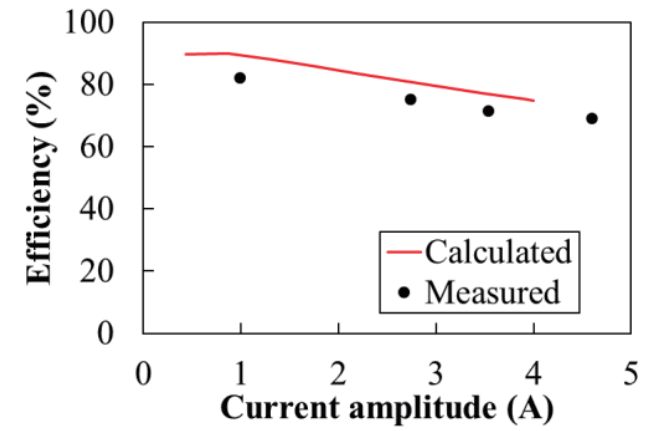

(a) Base model at rotational speed of $500 \mathrm{rpm}$

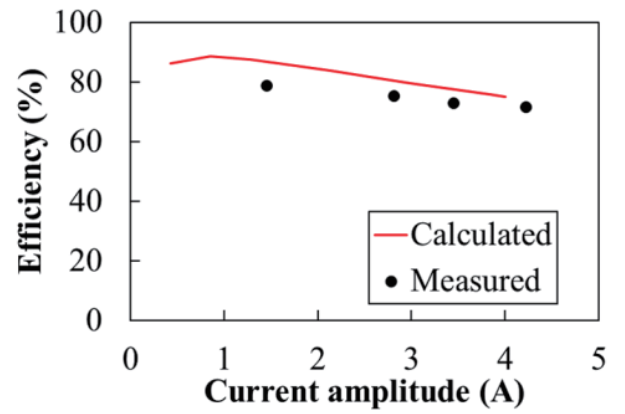

(b) Proposed model at rotational speed of $500 \mathrm{rpm}$

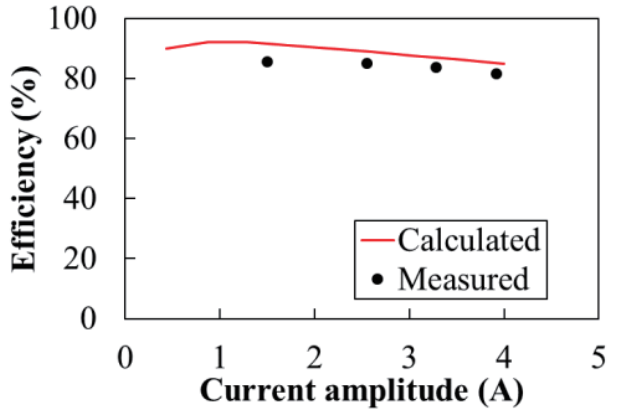

(c) Base model at rotational speed of $1000 \mathrm{rpm}$

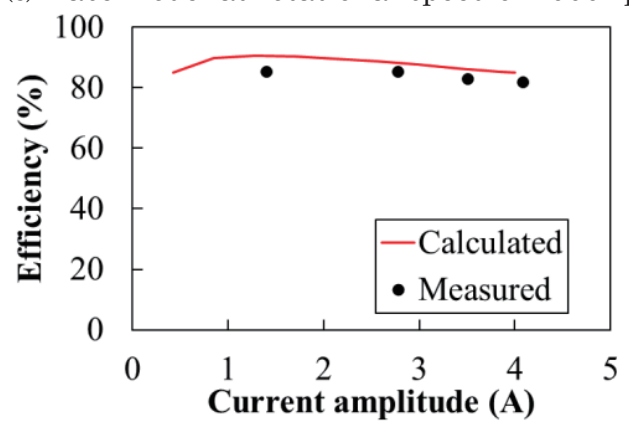

(d) Proposed model at rotational speed of $1000 \mathrm{rpm}$

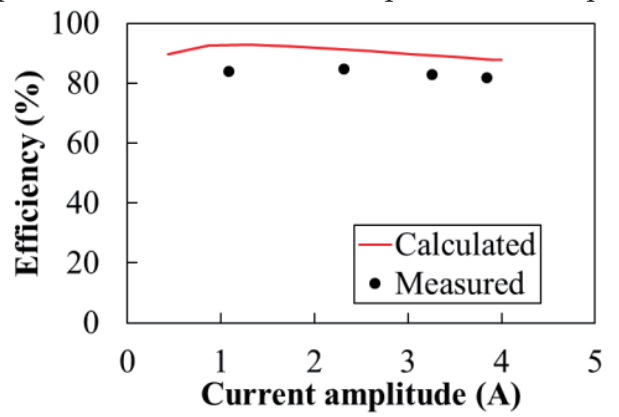

(e) Base model at rotational speed of $1300 \mathrm{rpm}$

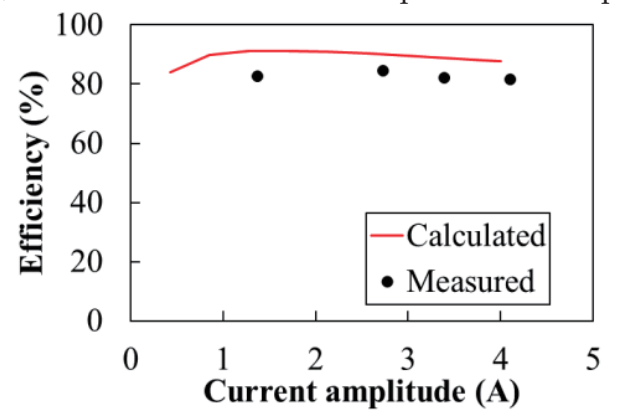

(f) Proposed model at rotational speed of $1300 \mathrm{rpm}$

Fig. 13. Relationship between current amplitude and efficiency 
The magnetic torque is indicated by the filled bars, and the reluctance torque is indicated by the hatched bars. It can be seen from this figure that the experimental value of the proposed model's magnetic torque is lower than the calculated value.

Fig. 13 shows the relationship between the current amplitude and the efficiency of the base and proposed models at rotation speeds of 500, 1000, and $1300 \mathrm{rpm}$. The calculated values are indicated by the solid lines, and the experimental values are indicated by the symbols. In the figures, the experimental values of efficiency were smaller than the calculated ones at all rotational speeds because the effect of the increase in iron loss due to harmonics and mechanical loss were not considered for the calculated values. The maximum efficiency of the measured values was $85.6 \%$ for the base model and $85.4 \%$ for the proposed model; the maximum efficiency of both models had almost the same value.

\section{Conclusion}

In this paper, we proposed an asymmetrical magnetic pole structure for an IPMSM using bonded neodymium magnets. We prototyped the proposed motor and compared the characteristics with a conventional IPMSM using sintered neodymium magnets. By adopting the asymmetrical magnet arrangement, the current phase angle, where the magnetic torque is maximized, could be set to almost the same phase by maximizing the reluctance torque.
Although the torque of the proposed motor was smaller than the conventional motor due to a problem with the dimensions of the bonded neodymium magnets, it was shown that this asymmetrical magnetic pole structure for IPMSMs using Dy-free bonded neodymium magnets could achieve performance equivalent to the conventional IPMSM using sintered neodymium magnets.

As future work, we plan to simplify the shapes of the magnets to prevent dimensional errors.

\section{References}

1) M. Sanada, Y. Inoue, and S. Morimoto: IEEJ Trans. on IA, 13, 1401 (2011).

2) Y. Yoshikawa, T. Ogawa, Y. Okada, S. Tsutsumi, H. Murakami, and S. Morimoto: IEEJ Trans. on IA, 136, 997 (2016).

3) S. Kusase and A. Shichijo: The Papers of Joint Technical Meeting on Motor Drive and Rotating Machinery IEEJ, MD-11-020, RM-11-041 (2011).

4) Y. Nara Y. Yoshida, and K. Tajima: The Papers of Technical Meeting on Magnetics IEEJ, MAG-17-003 (2017).

5) Xianxin Zeng, Li Quan, Xiaoyong Zhu, Lei Xu, and Fangjie Liu: IEEE Trans. on AS, 29, 0602704 (2019).

6) Fuzhen Xing, Wenliang Zhao, and Byung-ll Kwon: IET Electr. Power Appl., 13, 573 (2019).

7) Y. Yoshida, Y. Nara, and K. Tajima: The Papers of Technical Meeting on Rotating Machinery IEEJ, RM-17-121 (2017).

Received Oct. 31, 2019; Revised Dec. 4, 2019; Accepted Dec. 14, 2019 